\title{
Privacy preserving Intrusion Detection via Homomorphic Encryption
}

\author{
Luigi Coppolino, Salvatore D’Antonio, \\ Giovanni Mazzeo, Luigi Romano and Luigi Sgaglione \\ University of Naples "Parthenope" \\ Naples, Italy \\ \{luigi.coppolino, salvatore.dantonio, giovanni.mazzeo, \\ luigi.romano, luigi.sgaglione\} @ uniparthenope.it
}

\author{
Domenico Cotroneo, and Andrea Scognamiglio \\ University of Naples Federico II \\ Naples, Italy \\ cotroneo@unina.it, \\ andrea.scognamiglio6@studenti.unina.it
}

\begin{abstract}
In the recent years, we are assisting to an undiminished, and unlikely to stop number of cyber threats, that have increased the organizations/companies interest about security concerns. Further, the rising costs of an efficient IT security staff and environment is posing a significant challenge. These have created a new fast growing trend named Managed Security Services (MSS). Often customers turn to MSS providers to alleviate the pressures they face daily related to information security. One of the most critical aspect, related to the outsourcing of security issues, is privacy. Security monitoring and in general security services require access to as much data as possible, in order to provide an effective and reliable service. It is the well known conflict between privacy and security, a particularly evident problem in security monitoring solutions.

This paper analyzes a scenario of MSS in order to provide a privacy preserving solution that allows the security monitoring without violating the privacy requirements. The basic idea relies on the usage of the Homomorphic Encryption technology. Encrypting data using homomorphic schemes, cloud computing and MSS providers can perform different computations on encrypted data without ever having access to their decryption. This solution keeps data confidential and secured, not only during exchange and storage, but also during processing.

We provide an ad-hoc Intrusion Detection System architecture for privacy preserving security monitoring, considering as counter threats Code Injection attacks on homomorphically encrypted fields.
\end{abstract}

Index Terms-IDS, Managed Security Services, Homomorphic Encryption, GDPR, Privacy

\section{INTRODUCTION}

In the recent years, we are assisting to an undiminished, and unlikely to stop number of cyber threats with a total cost for the world of almost $\$ 600$ billion, or $0.8 \%$ of global GDP (Global gross Domestic Product) [1].

In particular, the adoption by the hacker of new technologies and the increased interest in the Cybercrime service have generated even more sophisticated threats. With the increase of

This project has received funding from the European Unions Horizon 2020 Framework Programme for Research and Innovation under grant agreements No 740712 (COMPACT) and "Aziende e tecnologie smart: modelli, misurazione delle performance, gestione della conoscenza e soluzioni tecnologiche" project under the "Ricerca Competitiva" programme funded by University of Naples "Parthenope". these and others malicious activities, the organizations/companies are even more interested about security concerns. Further, the rising costs of an efficient IT security staff and environment is posing a significant challenge. Such inflated IT budgets are continuously becoming a bottleneck for the companies across industries. These have created a new fast growing trend that outsources the security services to third parties. This trend takes the name of Managed Security Services (MSS). MSS are security services (remote management and monitoring of IT security) that have been outsourced to a service provider. The organizations are now considering the outsourcing of the IT security jobs to the MSS vendors in order to focus on their core business activities. The key factors with such an arrangement include effective management of IT security, seamless monitoring and cost-effectiveness [2] [3]. Companies turn to managed security services providers to alleviate the pressures they face daily related to information security such as targeted malware, customer data theft, skills shortages and resource constraints. Altogether, this market is expected to experience a high growth riding on the back of the increase in the cyber crime and its threats. Most organizations (74\%) manage IT security in-house, but $82 \%$ of IT professionals said they have either already partnered with, or planned to partner with, a managed security service provider [4]. One of the most critical aspects, related to the outsourcing of security issues, is the privacy. Security monitoring and in general security services require access to as much data as possible, in order to provide an effective and reliable service. They have the problem to fight threats of being exposed to malicious attackers and especially to deal with the well known conflict between security and privacy references. This conflict is a particularly evident problem in security monitoring solutions where the more information they collect and process, the more the security they guarantee and the less privacy they respect. On one side knowledge is the key element for security monitoring: detailed knowledge of a monitored system is a key factor for guaranteeing its security. On the other side, such a detailed knowledge includes many user's sensitive information that should be preserved for privacy purposes [5]. So the real problem, nowadays, is not finding a third party who monitors our security, but it is about trusting it. 
Another reason that drives research for innovative solutions is the incumbent duty to comply with the directives of the recent GPDR [6]. All MSS or Cloud Computing providers will have to consider new legal developments following the adoption of the new General Data Protection Regulation [7].

Our contribution is to seek new solutions and technologies to address the security-privacy trade off, allowing customers to take advantage of outsourced managed security services without giving third-party entities full access to their own sensitive information. In particular, this paper proposes a novel Intrusion Detection System (IDS) architecture that using Homomorphic Encryption allows a privacy preserving security monitoring. To the best of our knowledge this is the first tentative of using $\mathrm{HE}$ technology in a security monitoring system.

The remainder of this paper is organized as follows. In Section II an overview of related works is presented, in Section III we provide background of HE and IDS. Then, in Section IV the threat model is defined. Section V describe a typical IDs architecture and how this architecture evolves in order to allow a privacy preserving security monitoring. Finally, Section VI provides concluding remarks.

\section{RELATED WORK}

Many approachs are available in literature to guarantee the data privacy in MSS or cloud environment but no one is able to guarantee this when who processes the data cannot be considered as trusted or does not have authorization for the data processing. In [8] a set of security protocols for ensuring the privacy and legal compliance of customer data in cloud computing architectures are provided, allowing for the secure storage and processing of users' confidential data. The described approach is based on the tamper-proof facilities of cryptographic coprocessors in order to provide a secure execution domain in the computing cloud that is physically and logically protected from unauthorized access. This approach provides a trusted execution area in a cloud environment, advertising the user on different processing operations performed over the data. The problem of this approach is that it requires an additional resource the cryptographic coprocessor and that the processing entity is a trusted user. In [9] the authors propose a trust- and risk-aware access control framework for Threat Detection Systems, where each access request is evaluated by comparing the privacy-risk and the trustworthiness of the request. In [10], many techniques are investigated, including secure multiparty computation, and homomorphic encryption as possible solution for the privacy aware image processing in a cloud environment. In [11], the authors propose the use of homomorphic encryption to address risks related to malicious insiders in Smart Grid applications, in general, and for Wide Area Monitoring Systems, in particular.

\section{BACKGROUND}

\section{A. Homomorphic Encryption}

Conventional symmetric and asymmetric encryption techniques (as DES, RSA, AES, etc.) can't be useful as solutions to allow privacy preserving monitoring solution because they do not allow processing on encrypted data without decrypt it. Instead, Homomorphic Encryption (HE) is a form of encryption that allows computation on ciphertexts, generating an encrypted result which, when decrypted, matches the result of the operations as if they had been performed on the plaintext. $\mathrm{HE}$ relies on something like an asymmetric scheme, which requires two keys one to process (public key) and one to encrypt/decrypt (secret key) data, before and after processing phase. The service (untrusted) provider processes the received data encrypted with the homomorphic secret key by the end user, performs operations over this encrypted data using the public key and sends the encrypted result to the end user, which is the only one able to decrypt it (owner of the homomorphic secret key) (Fig. 1).

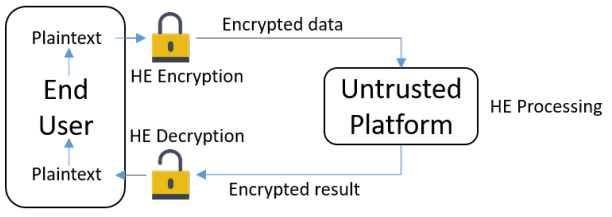

Fig. 1. HE scheme

The first HE algorithm, i.e., Partially Homomorphic Encryption (PHE) [12] [13], had the ability to carry out just one type of operations (e.g., addition, or multiplication). Clearly, the limitation in the type of executable computations hampered the usage of HE in practical contexts. Gentry et al. [14] provided the first implementation of a Fully Homomorphic Encryption (FHE) scheme. The security of the system is based on the noise introduced into the ciphered text. When the noise reaches some maximum amount, the ciphertext becomes undecryptable. In [15], the authors provide also a parallel version of the FHE for a cloud environment in order to improve its performance. An attempt to simplify the method has been provided by Van Dijk et al. [16] who proposed a Somewhat Homomorphic Encryption (SHE) over the integers. The price to pay with SHE is given by the limited number of mathematical operations that can be performed. However, in many real-world applications (e.g., medical, financial) this seems reasonable since as Naehrig et al. [17] analysis reports most of the evaluations required, i.e., one-time statistical functions, fits well with SHE constraints. An accurate list of potential real use cases that motivates the continuous improvements of the Homomorphic Encryption was curated during the Crypto Standardization Workshop [18].

To define computation on encrypted data, we must fix some model of computation. Supposing that plaintext space is $P=$ $\{0,1\}$, homomorphic scheme always use binary circuits with fan-in-2 gates to describe the functions we want to compute on encrypted data.

Definition 3.1: A homomorphic public-key encryption scheme $(\xi)$ consists of four procedures,

\section{$\xi=($ KeyGen, Encrypt, Decrypt, Evaluate $)$}

- KeyGen: generate public and secret keys; 
- Encrypt: encrypt plaintext in a ciphertext;

- Decrypt: decrypt a ciphertext in a plaintext;

- Evaluate: perform computing on encrypted data, executing the function described by the binary circuit.

For each binary circuit, it is important to denote his size (number of gates), his depth (longest input-to-output path) and his number of inputs.

Above all binary circuit's depth is fundamental, because the greater the depth, the greater the noise that affects data encrypted after the computing performed.

When the noise exceeds a certain threshold, performing the encrypted data decryption becomes impossible. At the current state of art, the only available solution is to use the bootstrap technique.

Definition 3.2:

Let $\xi=$ (KeyGen, Encrypt, Decrypt, Evaluate) be a homomorphic encryption scheme and $C=\left\{C_{\tau}\right\}_{\tau \in N}$ be some circuit family. $\xi$ is perfectly correct for $C$ if it correctly decrypts both fresh and evaluated ciphertexts.

This definition refers two different types of ciphertexts: fresh and evaluated. In fact exists a huge difference between fresh ciphertexts, referred to ciphertexts output by Encrypt function and hence not affected by any kind of noise, and evaluated ciphertexts, instead referred to ciphertexts output by Evaluate function and hence their noise grow according with the binary circuit's depth.

Although homomorphic cryptography may seem like the panacea of the cryptographic world, unfortunately it is not due to different limitations:

- Memory consumption and computation time overheads are still a major drawback of HE. For example, members of the Microsoft Research team implemented a Logistic Regression Model on encrypted data [19] using their homomorphic library SEAL [20]. Considering memory usage, $1 \mathrm{Mb}$ of data results in more than $10 \mathrm{~Gb}$ of encrypted data. As far as computation, addition is still manageable as it takes under $1 \mathrm{~ms}$. However, multiplication takes over 5 seconds.

- The output is encrypted and hence, no data interpretation is possible without the appropriate secret key.

- To be able to compute on encrypted data, inputs must be encrypted under the same conditions and with the same key.

- Due to his native hidden nature, homomorphic encryption can't allow to check if every computation was carried out as expected.

\section{B. Intrusion Detection System (IDS)}

An Intrusion Detection System (IDS) is a device or software application that monitors a network or systems for malicious activities or policy violations. An IDS works by monitoring system activity through examining vulnerabilities in the system, the integrity of files and conducting an analysis of patterns based on already known attacks.

An IDS can be classified according to the detection model:
- Signature-Based IDS refers to the detection of attacks by looking for specific patterns. This is useful for finding already known threats, but does not help in finding unknown threats, variants of threats or hidden threats, for which no pattern is available.

- Anomaly-Based IDS introduced to detect unknown attacks. The basic approach uses machine learning to create a model of trustworthy activity, and then compare new behavior against this model. It may suffer from false positives: previously unknown legitimate activity may also be classified as malicious.

Furthermore, an IDS can be classified by scope of protection:

- Network Intrusion Detection System (NIDS) placed at a strategic point or points within the network to monitor traffic to and from all devices on the network.

- Host Intrusion Detection System (HIDS) runs on individual hosts or devices on the network. A HIDS monitors the inbound and outbound packets from the device only and will alert the user or administrator if suspicious activity is detected.

\section{THREAT MODEL}

A threat model is essentially a structured representation of all the information that affects the security of an application. The threat model of this work focuses mainly on Injection Attacks, according to the OWASP Top Ten for 2010 document [21].

Injection is the exploitation of a computer bug that is caused by processing invalid data. Injection is used by an attacker to "inject" code into a vulnerable computer program and to change the course of execution or to provide additional effects. The result of successful code injection can be disastrous.

According to the threat model, a Signature-Based Intrusion Detection System has been taken as reference.

As discussed in the section III-A, Homomorphic Encryption has several limitations, one of them is the overhead.

Because of its overhead, HE is suitable for use to encrypt only low frequency data and contained in size, i.e., application data. Indeed if HE was used to encrypt all data generated by the monitored system (application and system data), an unmanageable overhead would be produced.

In particular, the HE solution should be used to preserve privacy of sensitive user information, which is often simple data fields of interest for human use and consumption.

It could happen that injection is performed by bots, thus still resulting in data volumes that can not be managed by using HE. Nevertheless this case would result in an anomalous user requests which would likely result in a alarm triggered by a rule not needing to analyze requests contents.

\section{IDS ARCHITECTURE}

\section{A. Common IDS Architecture}

Fig. 2 shows a simplified IDS architecture that has several fundamental components [22]: 


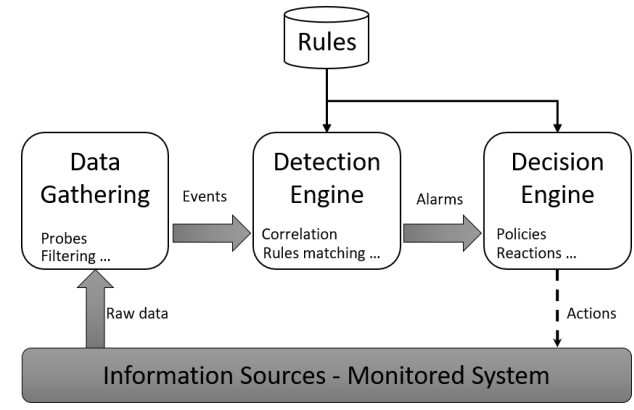

Fig. 2. Components of a Generic Intrusion Detection System

- Information Source - Monitored System - set of sources that generate data submitted to the Intrusion Detection System;

- Data Gathering - also called data collection, is the process of gathering or measuring information on targeted variables in an established system. This phase subsequently enables alerts detection to be performed on information collected from different sources;

- Detection Engine - is the business core of the whole system. Its function is to analyze data collected and match them with known attacks, expressed in the form of rules. These rules are stored in a database and eventually updated. If an attack does not have the equivalent rule (anomaly or signature based) in the database, it is an unknown attack and it can not be detected;

- Rules - set of known attacks expressed in formal format that allows detection engine to unambiguously identify some patterns in the data, so that it can detect any anomalies and generate alerts;

- Decision Engine - is alarmed and activated by the Detection engine, it uses a decision table to take an action or to send a report.

\section{B. Signature-Based IDS Architecture for privacy preserving security monitoring}

Before to describe the proposed IDS architecture we need to focus our attention on the selected threat model: Code Injection. In order to detect this kind of attack, considering a signature based approach, we need to define the signature that allows us to detect the injection of the malicious content (typically code: sql or script) inside normal information.

The chosen signature examines a single string field in order to check if it contains special characters, i.e., dots, semicolons, commas, brackets or any other non-alphanumeric characters in the ASCII table, which can lead back to an Injection Attack. The signature has an atomic structure: it examines a single string field for a match.

When the injection is applied to a sensitive string field, standard detection techniques cannot applied without a privacy violation. Instead using $\mathrm{HE}$, is it possible to process the sensitive string field in an encrypted form allowing the privacy preserving. In order to do that, the common IDS architecture must be specialized including on homomorphic encryption support and an homomorphic function must be defined.

In Fig. 3 is shown the specialized IDS architecture.

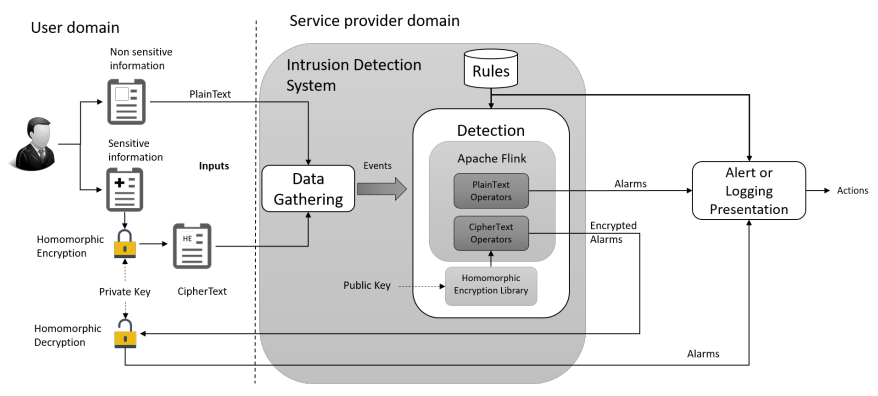

Fig. 3. IDS Architecture

The architecture is composed of several components:

- Inputs - inputs refer to two data types: plain data and encrypted data. Encrypted data are data homomorphically encrypted using appropriate libraries;

- Intrusion Detection System - composed by:

- Data Gathering - has the role to collect data by different input sources;

- Detection Engine - in its detection models uses operators on both plain and homomorphic encrypted data. These means that each homomorphic processing must have a corresponding homomorphic operator that relies on an homomorphic function (typically written in $\mathrm{C}++$ ).

- Alert or Logging Presentation - is the decision engine, its behavior varies depending on whether its inputs are plain or encrypted. If encrypted, its inputs must first be decrypted.

As shown in the architecture, the Alert and Logging phase, which is typically performed by the detection system, now is external, because the IDS that is an untrusted entity, does not have the secret key to decrypt the detection outcomes. So the monitoring results (when homomorphic data are involved) must be sent to the end user, who has the secret key, to allow him to decrypt the ciphertext. Once here, the end user will decide on actions to be taken.

\section{Implementation Details}

We focus on the application of Homomorphic Encryption using one of the best solutions: TFHE [23] an open-source library for fully homomorphic encryption, distributed under the terms of the Apache 2.0 license and maintained by the SPQlios team.

TFHE is a $\mathrm{C} / \mathrm{C}++$ library which implements a very fast gateby-gate bootstrapping based on [24] and [25].

We called "Check Special Chars" the function that allows to check if special characters (i.e., dots, semicolons, commas, brackets or any other non-alphanumeric characters) are present inside an homomorphically encrypted string field. From a practical point of view, this function checks if each string's character belongs to specific bounds defined by the ASCII 
codes for the characters allowed (a-Z, A-Z, 0-9). Assuming that the string has fixed length, i.e., 8 alphanumeric characters, the pseudo code of this function is the following:

$$
\begin{aligned}
& \text { Integer } 8 \text { a[8] } \\
& \text { Integer } 8 b, c, d, e, f, g, s \\
& \text { Integer } 8 m, n, o, p \\
& \text { Bit check }=1 \\
& b=\text { Integer } 8\left({ }^{\prime} a^{\prime}\right), c=\text { Integer } 8\left({ }^{\prime} z^{\prime}\right) \\
& d=\text { Integer } 8\left({ }^{\prime} A^{\prime}\right), e=\text { Integer } 8\left({ }^{\prime} Z^{\prime}\right) \\
& f=\text { Integer } 8\left({ }^{\prime} 0^{\prime}\right), g=\text { Integer } 8\left({ }^{\prime} 9^{\prime}\right) \\
& s=\text { Integer } 8\left({ }^{\prime}{ }^{\prime}\right) \\
& \text { for } i=0: 8 \\
& m=\overline{(a[i]<b)} \text { AND } \overline{(a[i]>c)} \\
& n=\overline{(a[i]<d)} A N D \overline{(a[i]>e)} \\
& o=\overline{(a[i]<f)} A N D \overline{(a[i]>g)} \\
& p=(a[i]==s) \\
& \text { check }=\text { check } A N D(m \text { OR } n \text { OR on } O R p)
\end{aligned}
$$

In this pseudo code, check is false if the string contains characters which are out of the bounds. It must be noted that:

- the provided pseudo code is only an high level representation of the homomorphic operator that requires two input parameters: the string to be tested and the range of normal characters, both homomorphically encrypted with the same secret key

- the comparison operators " $>=$ " and " $<=$ " have been implemented respectively as "not $<$ " and "not $>$ " this is because the not operation is less expensive than the equal one.

The homomorphic operator in its real form is a binary circuit that can be represented as a BLIF file (Berkeley Logic Interchange Format) that is a hardware description language designed for the hierarchical description of sequential circuits. By using ABC (a common System for Sequential Synthesis and Verification), we have analyzed this BLIF file in order to understand the complexity of the homomorphic operator, and we have that it is composed of:

- 112 inputs: 64 inputs for the string to test (8 inputs per character) and 8 inputs for each "valid" character $(\mathrm{a}, \mathrm{Z}, \mathrm{A}, \mathrm{Z}, 0,9)$.

- 1 output, the true/false value of the check function.

- 1860 gates: 991 and, 109 inverter and 760 others (nand, nor, mux, etc).

- 15 levels deep.

In the following listing is reported the code used to implement the checkSpecialChars operator by using TFHE. The range used to identify the special chars "specialChars" passed as input to this function is the following homomorphical encrypted string: "azAZ09" (this string must be encrypted with the same private key used to encrypt the string to be analyzed - op1).

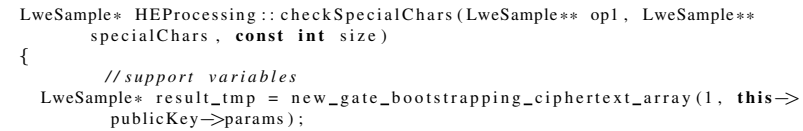

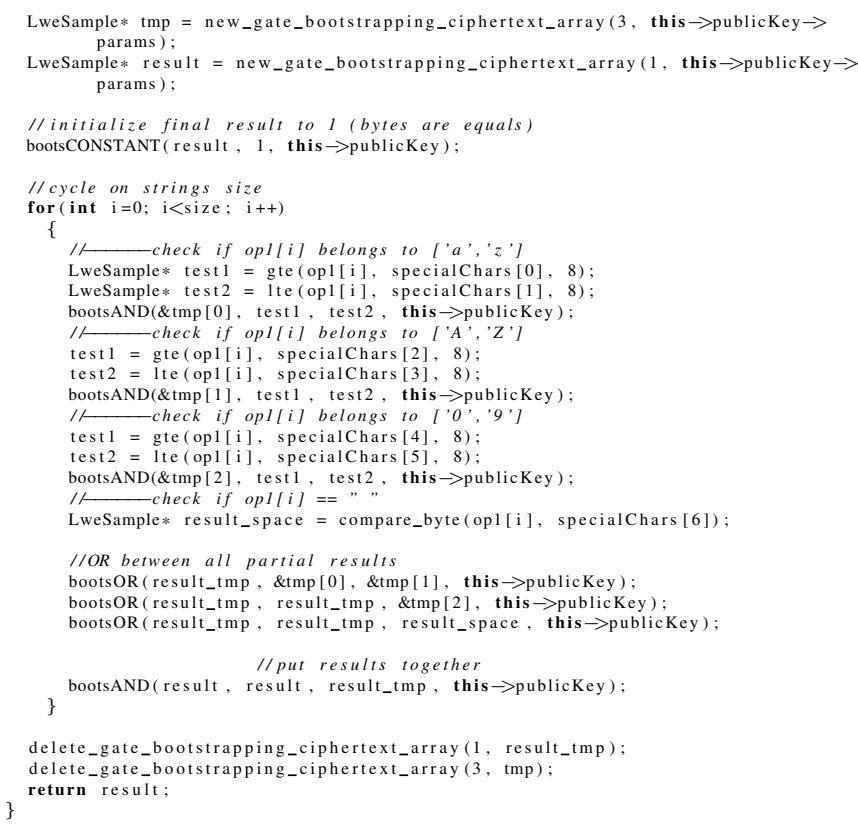

The functions "gte" and "lte" are implemented by using a bit by bit comparison based on the TFHE XOR and MUX operators.

\section{Evaluation}

In order to test the effectiveness and the performance of the proposed approach, by using an Ubuntu 16.04 LTS virtual environment with $6 \mathrm{~GB}$ of RAM and 1 processor, we have tested two typical examples of code injection (XSS) inside a string value containing personal information (name and surname). The first malicious code steals the session cookie of an authenticated user, while the second one redirects the victim towards the attackers web server. The results of the tests are reported in Table I.

TABLE I

EVALUATION RESULTS

\begin{tabular}{|c|c|}
\hline \multicolumn{2}{|c|}{ XSS } \\
\hline Detection result & Response time \\
\hline \multicolumn{2}{|c|}{ "john doe" } \\
\hline Secure & $\sim 12 \mathrm{sec}$ \\
\hline \multicolumn{2}{|c|}{ "john $<$ script $>$ alert(document.cookie) $</$ script $>$ doe" } \\
\hline Not Secure & $\sim 73 \mathrm{sec}$ \\
\hline "john $<$ script src $=$ "http://devilhost:8090/script.js" $></$ script $>$ doe" \\
\hline Not Secure & $\sim 105 \mathrm{sec}$ \\
\hline
\end{tabular}

Looking at the results it is worth noting that by using HE it is possible to monitor the code injection attack without accessing the unencrypted version of the data, but paying a big cost in terms of time overhead that increases with the length of the data to be checked. This makes our approach applicable only in domains where the service response time can afford the additional processing time required by the homomorphic processing [18] or in general any other service where the response time is at least soft-realtime like information sharing services (i.e. Medical Patient Summary Exchange, Public Administration security information exchange, etc.). 


\section{CONCLUSION AND FUTURE WORK}

In this paper we present a Signature-Based IDS Architecture for privacy preserving security monitoring using a homomorphic encryption technique. It allows a customer to trust in third-party entities, because he is confident that his sensitive data will remain encrypted during exchange, storage and processing, by means of homomorphic encryption.

Preprint

\section{REFERENCES}

[1] M. CSIC, "Economic impact of cybercrime," Center for Strategic and International Studies, Report 2018, 2018, https://www.csis.org/analysis/economic-impact-cybercrime.

[2] F. Amato, F. Moscato, V. Moscato, and F. Colace, "Improving security in cloud by formal modeling of iaas resources," Future Generation Computer Systems, vol. 87, pp. 754 - 764, 2018. [Online]. Available: http://www.sciencedirect.com/science/article/pii/S0167739X17305964

[3] F. Amato, N. Mazzocca, and F. Moscato, "Model driven design and evaluation of security level in orchestrated cloud services," Journal of Network and Computer Applications, vol. 106, pp. $78-89,2018 . \quad$ [Online]. Available: http://www.sciencedirect.com/science/article/pii/S1084804517304022

[4] OUT-LOW.com, "Pressure to deliver new it projects despite security concerns felt by $80 \%$ of it professionals," https://www.outlaw.com/articles/2014/february/pressure-to-deliver-new-it-projectsdespite-security-concerns-felt-by-80-of-it-professionals/, 2014 , accessed: 2018-10-24

[5] G. Cerullo, G. Mazzeo, G. Papale, B. Ragucci, and L. Sgaglione, "Chapter 4 - iot and sensor networks security," in Security and Resilience in Intelligent Data-Centric Systems and Communication Networks, ser. Intelligent Data-Centric Systems, M. Ficco and F. Palmieri, Eds. Academic Press, 2018, pp. 77 - 101. [Online]. Available: http://www.sciencedirect.com/science/article/pii/B9780128113738000045

[6] EC. (2018) General data protection regulation. [Online]. Available: https://ec.europa.eu/info/law/lawtopic/dataprotection/dataprotectioneu_en

[7] L. Sgaglione and G. Mazzeo, "A gdpr-compliant approach to realtime processing of sensitive data," in Intelligent Interactive Multimedia Systems and Services, G. De Pietro, L. Gallo, R. J. Howlett, L. C. Jain, and L. Vlacic, Eds. Cham: Springer International Publishing, 2019, pp. 43-52.

[8] W. Itani, A. Kayssi, and A. Chehab, "Privacy as a service: Privacy-aware data storage and processing in cloud computing architectures," in 2009 Eighth IEEE International Conference on Dependable, Autonomic and Secure Computing, Dec 2009, pp. 711-716.

[9] N. Metoui, M. Bezzi, and A. Armando, "Trust and risk-based access control for privacy preserving threat detection systems," in Future Data and Security Engineering, T. K. Dang, R. Wagner, J. Küng, N. Thoai, M. Takizawa, and E. Neuhold, Eds. Cham: Springer International Publishing, 2016, pp. 285-304.

[10] Z. Qin, J. Weng, Y. Cui, and K. Ren, "Privacy-preserving image processing in the cloud," IEEE Cloud Computing, pp. 1-1, 2018.

[11] L. Coppolino, S. D'Antonio, G. Mazzeo, L. Romano, and L. Sgaglione, "An approach for securing cloud-based wide area monitoring of smart grid systems," in Advances in Network-Based Information Systems, L. Barolli, N. Kryvinska, T. Enokido, and M. Takizawa, Eds. Cham: Springer International Publishing, 2019, pp. 952-959.

[12] T. El Gamal, "A public key cryptosystem and a signature scheme based on discrete logarithms," in Proceedings of CRYPTO 84 on Advances in Cryptology. New York, NY, USA: SpringerVerlag New York, Inc., 1985, pp. 10-18. [Online]. Available: http://dl.acm.org/citation.cfm?id=19478.19480

[13] P. Paillier, "Public-key cryptosystems based on composite degree residuosity classes," in Advances in Cryptology - EUROCRYPT '99, J. Stern, Ed. Berlin, Heidelberg: Springer Berlin Heidelberg, 1999, pp. 223-238.

[14] C. Gentry, "Fully homomorphic encryption using ideal lattices," in Proceedings of the Forty-first Annual ACM Symposium on Theory of Computing, ser. STOC '09. New York, NY, USA: ACM, 2009, pp. 169178. [Online]. Available: http://doi.acm.org/10.1145/1536414.1536440
[15] R. Hayward and C.-C. Chiang, "Parallelizing fully homomorphic encryption for a cloud environment," Journal of Applied Research and Technology, vol. 13, no. 2, pp. 245 - 252, 2015. [Online]. Available: http://www.sciencedirect.com/science/article/pii/S166564231500005X

[16] M. van Dijk, C. Gentry, S. Halevi, and V. Vaikuntanathan, "Fully homomorphic encryption over the integers," in Advances in Cryptology - EUROCRYPT 2010, H. Gilbert, Ed. Berlin, Heidelberg: Springer Berlin Heidelberg, 2010, pp. 24-43.

[17] M. Naehrig, K. Lauter, and V. Vaikuntanathan, "Can homomorphic encryption be practical?" in Proceedings of the 3rd ACM Workshop on Cloud Computing Security Workshop, ser. CCSW '11. New York, NY, USA: ACM, 2011, pp. 113-124. [Online]. Available: http://doi.acm.org/10.1145/2046660.2046682

[18] D. Archer, L. Chen, J. H. Cheon, R. Gilad-Bachrach, R. A. Hallman, Z. Huang, X. Jiang, R. Kumaresan, B. A. Malin, H. Sofia, Y. Song, and S. Wang, "Applications of homomorphic encryption," HomomorphicEncryption.org, Redmond WA, Tech. Rep., July 2017.

[19] H. Chen, R. Gilad-Bachrach, K. Han, Z. Huang, A. Jalali, K. Laine, and K. Lauter, "Logistic regression over encrypted data from fully homomorphic encryption," BMC Medical Genomics, vol. 11, no. 4, p. 81, Oct 2018. [Online]. Available: https://doi.org/10.1186/s12920018-0397-z

[20] "Simple Encrypted Arithmetic Library (release 3.1.0)," https://github.com/Microsoft/SEAL, Dec. 2018, microsoft Research, Redmond, WA

[21] O. W. A. S. Project. (2019) Owasp top ten. [Online]. Available: https://www.owasp.org/index.php/Category:OWASP_Top_Ten_Project

[22] H. Jahankhani, Handbook of electronic security and digital forensics. New Jersey: World Scientific, 2010.

[23] S. team. (2019) Fast fully homomorphic encryption over the torus. [Online]. Available: https://tfhe.github.io/tfhe/

[24] I. Chillotti, N. Gama, M. Georgieva, and M. Izabachène, "Faster fully homomorphic encryption: Bootstrapping in less than 0.1 seconds," in Advances in Cryptology - ASIACRYPT 2016, J. H. Cheon and T. Takagi, Eds. Berlin, Heidelberg: Springer Berlin Heidelberg, 2016, pp. 3-33.

[25] — "Improving the: faster packed homomorphic operations and efficient circuit bootstrapping," IACR Cryptology ePrint Archive, vol. 2017, p. 430, 2017. 